\title{
Synergy of shotgun-MS and 2DE for analysis of proteome heterogeneity
}

\author{
O. Kiseleva*, E. Poverennaya, S. Naryzhny, V. Zgoda, A. Lisitsa \\ Institute of Biomedical Chemistry, Moscow, Russia \\ *e-mail: olly.kiseleva@gmail.com
}

Key words: proteomics, proteoforms, proteome heterogeneity, shotgun MS, 2DE

Motivation and Aim: Proteome heterogeneity is an unavoidable factor that complicates proteomic studies. Necessity for investigation of such events as alternative splicing, single amino acid polymorphisms and posttranslational modifications at protein level is determined by significant influence of these events on expression and functional properties of proteins. Careful analysis of proteome, which takes into account aberrant proteoforms, provides a basis for understanding the machinery of complex biochemical systems.

Methods and Algorithms: Basing on the results of RNASeq analysis of HepG2 cell line, we created a transcriptomespecific library, containing 52 thousand protein sequences, encoded by 12 thousand genes. Such library allows to focus on individual variations and helps to avoid uncontrolled extension of search area by populational data, thus reducing FDR. One of the most popular methods of proteomic analysis - shotgun mass spectrometry - is characterized by low (ca. $20 \%$ ) coverage of protein sequence. Short peptides, detected with shotgun-MS, often do not allow to distinguish highly homologous proteoforms. To empower shotgun approach, we added two dimensional electrophoresis (2DE). After 2DE fractioning we cut the gel in 96 cells and analyzed every cell by MS. Fractioning of protein mixture before MS allows to enrich the results with coordinates of proteoform on the gel ( $\mathrm{p} I$ and $\mathrm{MW})$.

Results: 2DE profiling with further MS analysis allowed to discover over 2358 proteoforms (1658 of which were canonical, 224 - splice-forms, and 115 - with amino acid polymorphisms) encoded by 1904 genes. Without 2DE we identified only 925 proteoforms because of sample heterogeneity and lack of knowledge of physicalchemical parameters of proteoforms, used for specification of certain proteoform.

Conclusion: Effective tandem of 2DE/MS allows us to forecast modifications, which can change physicalchemical parameters (and the location of protein spot on the gel, consequently). Obtained results consist not only of evaluation of proteoforms implemented at the protein level, but also of improvement of experimental approaches to cell proteotyping.

Acknowledgements: We acknowledge the Leading Scientific School of Prof. Andrey Lisitsa (grant the Russian Federation of President NSh6313.2018.4). 